DOI 10.7603/s40956-016-0009-x

Moroccan J. Pure and Appl. Anal.(MJPAA)

RESEARCH ARTICLE

Volume 2(2), 2016, Pages 107-117

ISSN: $2351-8227$

\title{
New Hermite Hadamard type inequalities for twice differentiable convex mappings via Green function and applications
}

\author{
SAMET ERDEN ${ }^{a}$ AND MEHMET ZEKI SARIKAYA ${ }^{b}$
}

\begin{abstract}
We derive some Hermite Hamamard type integral inequalities for functions whose second derivatives absolute value are convex. Some eror estimates for the trapezoidal formula are obtained. Finally, some natural applications to special means of real numbers are given.

2010 Mathematics Subject Classification. 26D15, 41A55, 52A41.

Key words and phrases. Hermite-Hadamard inequality, convex function, Green function, Hölder inequality, Trapezoidal formula, special means.
\end{abstract}

\section{Introduction}

The following inequality is well known in the literature as the Hermite-Hadamard integral inequality (see, [2], [5]):

$$
f\left(\frac{a+b}{2}\right) \leq \frac{1}{b-a} \int_{a}^{b} f(x) d x \leq \frac{f(a)+f(b)}{2}
$$

where $f: I \subset \mathbb{R} \rightarrow \mathbb{R}$ is a convex function on the interval $I$ of real numbers and $a, b \in I$ with $a<b$. A function $f:[a, b] \subset \mathbb{R} \rightarrow \mathbb{R}$, is said to be convex if the following inequality holds

$$
f(\lambda x+(1-\lambda) y) \leq \lambda f(x)+(1-\lambda) f(y)
$$

for all $x, y \in[a, b]$ and $\lambda \in[0,1]$. We say that $f$ is concave if $(-f)$ is convex.

The inequalities (1) have grown into a significant pillar for mathematical analysis and optimization, besides, by looking into a variety of settings, these inequalities are

Received March 15, 2016 - Accepted June 20, 2016.

(C)The Author(s) 2016. This article is published with open access by Sidi Mohamed Ben Abdallah University

${ }^{a}$ Department of Mathematics, Faculty of Science, Bartın University, BARTIN-TURKEY

e-mail: erdensmt@gmail.com

${ }^{b}$ Department of Mathematics, Faculty of Science and Arts, Düzce University, Konuralp Campus, Düzce-TURKEY

e-mail: sarikayamz@gmail.com 
found to have a number of uses. What is more, for a specific choice of the function $\mathrm{f}$, many inequalities with special means are obtainable. Hermite Hadamard's inequality (1), for example, is significant in its rich geometry and hence there are many studies on it to demonstrate its new proofs, refinements, extensions and generalizations. You can check ([1], [2], [4], [5] and [10]-[15]) and the references included there.

In [1], Dragomir and Agarwal proved the following inequality connected with the right part of (1).

Theorem 1.1. Let $f: I^{\circ} \subseteq \mathbb{R} \rightarrow \mathbb{R}$ be a differentiable mapping on $I^{\circ}, a, b \in I^{\circ}$ with $a<b$. If $\left|f^{\prime}\right|$ is convex on $[a, b]$, then the following inequality holds:

$$
\left|\frac{f(a)+f(b)}{2}-\frac{1}{b-a} \int_{a}^{b} f(x) d x\right| \leq \frac{(b-a)}{8}\left(\left|f^{\prime}(a)\right|+\left|f^{\prime}(b)\right|\right) .
$$

In [6], Pearce and Pěcarić proved the following inequality.

Theorem 1.2. Let $f: I^{\circ} \subseteq \mathbb{R} \rightarrow \mathbb{R}$ be a differentiable mapping on $I^{\circ}, a, b \in I^{\circ}$ with $a<b$. If $\left|f^{\prime}\right|^{q}$ is convex on $[a, b]$, for some $q \geq 1$, then we have

$$
\left|\frac{f(a)+f(b)}{2}-\frac{1}{b-a} \int_{a}^{b} f(x) d x\right| \leq \frac{b-a}{4}\left(\frac{\left|f^{\prime}(a)\right|^{q}+\left|f^{\prime}(b)\right|^{q}}{2}\right)^{\frac{1}{q}} .
$$

In [8], Sarikaya and Aktan established some inequalities related to Hermite-Hadamard's inequality for functions whose second derivatives are convex.

Theorem 1.3. Let $f: I^{\circ} \subseteq \mathbb{R} \rightarrow \mathbb{R}$ be a differentiable mapping on $I^{\circ}$ such that $f^{\prime \prime} \in L[a, b]$ and $0 \leq \lambda \leq 1$ where $a, b \in I^{\circ}$ with $a<b$. If $\left|f^{\prime \prime}\right|$ is a convex on $[a, b]$, then the following inequalities hold:

$$
\begin{aligned}
& \left|(\lambda-1) f\left(\frac{a+b}{2}\right)-\lambda \frac{f(a)+f(b)}{2}+\frac{1}{b-a} \int_{a}^{b} f(x) d x\right| \\
& \leq \begin{cases}\frac{(b-a)^{2}}{12}\left[\left(\lambda^{4}+(1+\lambda)(1-\lambda)^{3}+\frac{5 \lambda-3}{4}\right)\left|f^{\prime}(a)\right|\right. & \\
\left.+\left(\lambda^{4}+(2-\lambda) \lambda^{3}+\frac{1-3 \lambda}{4}\right)\left|f^{\prime}(b)\right|\right], & \text { for } 0 \leq \lambda \leq \frac{1}{2} \\
\frac{(b-a)^{2}(3 \lambda-1)}{48}\left[\left|f^{\prime}(a)\right|+\left|f^{\prime}(b)\right|\right], & \text { for } \frac{1}{2} \leq \lambda \leq 1 .\end{cases}
\end{aligned}
$$

Remark 1.1. Under the assumptions Theorem 1.3 with $\lambda=1$ in Theorem 1.3, then we have

$$
\left|\frac{1}{b-a} \int_{a}^{b} f(x) d x-\frac{f(a)+f(b)}{2}\right| \leq \frac{(b-a)^{2}}{12}\left(\frac{\left|f^{\prime \prime}(a)\right|+\left|f^{\prime \prime}(b)\right|}{2}\right) .
$$

In [3], Husain et al. proved some inequalities related to Hermite-Hadamard's inequality for s-convex functions:

Theorem 1.4. Let $f: I^{\circ} \subseteq \mathbb{R} \rightarrow \mathbb{R}$ be a twice differentiable mapping on $I^{\circ}$ such that $f^{\prime \prime} \in L[a, b]$ where $a, b \in I^{\circ}$ with $a<b$. If $\left|f^{\prime \prime}\right|^{q}$ is $s$-convex on $[a, b]$ for some fixed 
$s \in(0,1]$ and $q \geq 1$, then the following inequality holds:

$$
\left|\frac{f(a)+f(b)}{2}-\frac{1}{b-a} \int_{a}^{b} f(x) d x\right| \leq \frac{(b-a)^{2}}{2 \times 6^{\frac{1}{p}}}\left[\frac{\left|f^{\prime \prime}(a)\right|^{q}+\left|f^{\prime \prime}(b)\right|^{q}}{(s+2)(s+3)}\right]^{\frac{1}{q}}
$$

where $\frac{1}{p}+\frac{1}{q}=1$.

Remark 1.2. If we take $s=1$ in (3), then we have

$$
\left|\frac{f(a)+f(b)}{2}-\frac{1}{b-a} \int_{a}^{b} f(x) d x\right| \leq \frac{(b-a)^{2}}{12}\left[\frac{\left|f^{\prime \prime}(a)\right|^{q}+\left|f^{\prime \prime}(b)\right|^{q}}{2}\right]^{\frac{1}{q}} \text {. }
$$

The interested reader is refer to [3],[7]-[9] for inequalities involving functions whose second derivatives are convex.

In this study, using functions whose second derivatives absolute values are convex, we establish new inequalities that are connected with the right-hand side of HermiteHadamard inequality. Then we give some eror estimates for trapezoidal quadrature formula by using these inequalities. Finally, we obtain some applications of these inequalities for special means are provided.

\section{Main Results}

In order to prove our main results we need the following lemma:

Lemma 2.1. Let $f: I \subset \mathbb{R} \rightarrow \mathbb{R}$ be a twice differentiable function on $I^{\circ}$ such that $f^{\prime \prime} \in L[a, b]$, the interior of the interval $I$, where $a, b \in I^{\circ}$ with $a<b$. Then the following identity holds:

$$
\frac{1}{b-a} \int_{a}^{b} \int_{a}^{b} G(x, y) f^{\prime \prime}(y) d y d x=\frac{1}{b-a} \int_{a}^{b} f(x) d x-\frac{f(a)+f(b)}{2}
$$

where

$$
G(x, y):= \begin{cases}\frac{(x-a)(y-b)}{(b-a)}, & a \leq x \leq y \leq b \\ \frac{(x-b)(y-a)}{(b-a)}, & a \leq y \leq x \leq b .\end{cases}
$$

Proof. By integration by parts, we have the following identity:

$$
\int_{a}^{b} G(x, y) f^{\prime \prime}(y) d y=\left.G(x, y) f^{\prime}(y)\right|_{y=a} ^{b} d x-\int_{a}^{b} \frac{\partial G(x, y)}{\partial y} f^{\prime}(y) d y d x .
$$

Because of $G(x, b)=G(x, a)=0$, we get

$$
\int_{a}^{b} G(x, y) f^{\prime \prime}(y) d y=-\frac{x-b}{b-a} \int_{a}^{x} f^{\prime}(y) d y-\frac{x-a}{b-a} \int_{x}^{b} f^{\prime}(y) d y
$$




$$
=f(x)-\frac{b-x}{b-a} f(a)-\frac{x-a}{b-a} f(b) .
$$

Integrating both sides of (5) with respect to $x$ over $[a, b]$ and rewritten, we have

$$
\int_{a}^{b} \int_{a}^{b} G(x, y) f^{\prime \prime}(y) d y d x=\int_{a}^{b} f(x) d x-(b-a) \frac{f(a)+f(b)}{2} .
$$

Hence, the proof is completed.

We obtain trapezoid inequality by using convexity of $\left|f^{\prime \prime}\right|$.

Theorem 2.1. Let $f: I^{\circ} \subseteq \mathbb{R} \rightarrow \mathbb{R}$ be a twice differentiable mapping on $I^{\circ}, a, b \in I^{\circ}$ with $a<b$. If $\left|f^{\prime \prime}\right|$ is convex on $[a, b]$, then, for all $y \in[a, b]$, the following inequality holds:

$$
\left|\frac{1}{b-a} \int_{a}^{b} f(x) d x-\frac{f(a)+f(b)}{2}\right| \leq \frac{(b-a)^{2}}{12}\left(\frac{\left|f^{\prime \prime}(a)\right|+\left|f^{\prime \prime}(b)\right|}{2}\right) .
$$

Proof. We take absolute value of (4), we find that

$$
\begin{aligned}
& \left|\frac{1}{b-a} \int_{a}^{b} f(x) d x-\frac{f(a)+f(b)}{2}\right| \\
\leq & \frac{1}{b-a} \int_{a}^{b} \int_{a}^{b}|G(x, y)|\left|f^{\prime \prime}(y)\right| d y d x \\
= & \int_{a}^{b} \int_{a}^{x} \frac{(b-x)(y-a)}{(b-a)^{2}}\left|f^{\prime \prime}(y)\right| d y d x+\int_{a}^{b} \int_{x}^{b} \frac{(b-y)(x-a)}{(b-a)^{2}}\left|f^{\prime \prime}(y)\right| d y d x .
\end{aligned}
$$

Since $\left|f^{\prime \prime}(y)\right|$ is convex on $[a, b]$, we have

$$
\left|f^{\prime \prime}\left(\frac{b-y}{b-a} a+\frac{y-a}{b-a} b\right)\right| \leq \frac{b-y}{b-a}\left|f^{\prime \prime}(a)\right|+\frac{y-a}{b-a}\left|f^{\prime \prime}(b)\right| .
$$

From (7), it follows that

$$
\begin{aligned}
& \left|\frac{1}{b-a} \int_{a}^{b} f(x) d x-\frac{f(a)+f(b)}{2}\right| \\
\leq & \left|f^{\prime \prime}(a)\right| \int_{a}^{b} \int_{a}^{x} \frac{(b-x)(y-a)(b-y)}{(b-a)^{3}} d y d x+\left|f^{\prime \prime}(b)\right| \int_{a}^{b} \int_{a}^{x} \frac{(b-x)(y-a)^{2}}{(b-a)^{3}} d y d x \\
& +\left|f^{\prime \prime}(a)\right| \int_{a}^{b} \int_{x}^{b} \frac{(x-a)(b-y)^{2}}{(b-a)^{3}} d y d x+\left|f^{\prime \prime}(b)\right| \int_{a}^{b} \int_{x}^{b} \frac{(x-a)(b-y)(y-a)}{(b-a)^{3}} d y d x .
\end{aligned}
$$


If we calculate the above integrals and also use elementary analysis, then we easily deduce required inequality (6) which completes the proof.

We derive trapezoid inequality by using convexity of $\left|f^{\prime \prime}\right|^{q}$.

Theorem 2.2. Let $f: I^{\circ} \subseteq \mathbb{R} \rightarrow \mathbb{R}$ be a twice differentiable mapping on $I^{\circ}, a, b \in I^{\circ}$ with $a<b$. If $\left|f^{\prime \prime}\right|^{q}$ is convex on $[a, b], q>1$, for all $y \in[a, b]$, then the following inequality holds:

$$
\begin{aligned}
& \left|\frac{1}{b-a} \int_{a}^{b} f(x) d x-\frac{f(a)+f(b)}{2}\right| \\
\leq & \frac{(b-a)^{2}}{(p+1)^{\frac{1}{p}}}\left(\frac{\left|f^{\prime \prime}(a)\right|^{q}+\left|f^{\prime \prime}(b)\right|^{q}}{2}\right)^{\frac{1}{q}}[B(p+2, p+1)+B(p+1, p+2)]^{\frac{1}{p}}
\end{aligned}
$$

where $\frac{1}{p}+\frac{1}{q}=1$ and $B(p, q)$ is Euler's Beta function.

Proof. We take absolute value of (4). Using Holder's inequality, we find that

$$
\begin{aligned}
& \left|\frac{1}{b-a} \int_{a}^{b} f(x) d x-\frac{f(a)+f(b)}{2}\right| \\
\leq & \frac{1}{b-a} \int_{a}^{b} \int_{a}^{b}|G(x, y)|\left|f^{\prime \prime}(y)\right| d y d x \\
\leq & \frac{1}{b-a}\left(\int_{a}^{b} \int_{a}^{b}|G(x, y)|^{p} d y d x\right)^{\frac{1}{p}}\left(\int_{a}^{b} \int_{a}^{b}\left|f^{\prime \prime}(y)\right|^{q} d y d x\right)^{\frac{1}{q}} .
\end{aligned}
$$

We need to calculate above integrals to prove the theorem.

Let's calculate first integral

$$
\begin{aligned}
& \int_{a}^{b} \int_{a}^{b}|G(x, y)|^{p} d y d x \\
= & \int_{a}^{b} \int_{a}^{x} \frac{(b-x)^{p}(y-a)^{p}}{(b-a)^{p}} d y d x+\int_{a}^{b} \int_{x}^{b} \frac{(b-y)^{p}(x-a)^{p}}{(b-a)^{p}} d y d x \\
= & \int_{a}^{b} \frac{(b-x)^{p}(x-a)^{p+1}}{(b-a)^{p}(p+1)} d x+\int_{a}^{b} \frac{(x-a)^{p}(b-x)^{p+1}}{(b-a)^{p}(p+1)} d x .
\end{aligned}
$$


Using the change of the variables $x-a=(b-a) u$ and $b-x=(b-a) u$ for the above integrals, respectively, we write

$$
\int_{a}^{b} \int_{a}^{b}|G(x, y)|^{p} d y d x=\frac{(b-a)^{p+2}}{p+1}[B(p+2, p+1)+B(p+1, p+2)]
$$

where $B(p, q)=\int_{0}^{1} u^{p-1}(1-u)^{q-1} d u,(p, q>0)$.

Since $\left|f^{\prime \prime}(t)\right|^{q}$ is convex on $[a, b]$, we have

$$
\left|f^{\prime \prime}\left(\frac{b-y}{b-a} a+\frac{y-a}{b-a} b\right)\right|^{q} \leq \frac{b-y}{b-a}\left|f^{\prime \prime}(a)\right|^{q}+\frac{y-a}{b-a}\left|f^{\prime \prime}(b)\right|^{q} .
$$

From (11), it follows that

$$
\begin{aligned}
& \int_{a}^{b} \int_{a}^{b}\left|f^{\prime \prime}(y)\right|^{q} d y d x \\
\leq & \left|f^{\prime \prime}(a)\right|^{q} \int_{a}^{b} \int_{a}^{b} \frac{b-y}{b-a} d y d x+\left|f^{\prime \prime}(b)\right|^{q} \int_{a}^{b} \int_{a}^{b} \frac{y-a}{b-a} d y d x \\
= & \frac{\left|f^{\prime \prime}(a)\right|^{q}+\left|f^{\prime \prime}(b)\right|^{q}}{2}(b-a)^{2} .
\end{aligned}
$$

If we substitute (10) and (12) in (9), then we easily deduce required inequality (8) which completes the proof.

Now, we establish trapezoid inequality in a different way by using convexity of $\left|f^{\prime \prime}\right|^{q}$.

Theorem 2.3. Let $f: I^{\circ} \subseteq \mathbb{R} \rightarrow \mathbb{R}$ be a twice differentiable mapping on $I^{\circ}, a, b \in I^{\circ}$ with $a<b$. If $\left|f^{\prime \prime}\right|^{q}$ is convex on $[a, b], q>1$, for all $y \in[a, b]$, then the following inequality holds:

$$
\begin{aligned}
& \left|\frac{1}{b-a} \int_{a}^{b} f(x) d x-\frac{f(a)+f(b)}{2}\right| \\
\leq & \frac{(b-a)^{2+\frac{1}{q}}}{(p+1)^{\frac{1}{p}} 2^{\frac{1}{q}}}\left\{[B(p+2, p+1)]^{\frac{1}{p}}\left(\frac{2\left|f^{\prime \prime}(a)\right|^{q}+\left|f^{\prime \prime}(b)\right|^{q}}{3}\right)^{\frac{1}{q}}\right. \\
& \left.+[B(p+1, p+2)]^{\frac{1}{p}}\left(\frac{\left|f^{\prime \prime}(a)\right|^{q}+2\left|f^{\prime \prime}(b)\right|^{q}}{3}\right)^{\frac{1}{q}}\right\}
\end{aligned}
$$

where $\frac{1}{p}+\frac{1}{q}=1$ and $B(p, q)$ is Euler's Beta function. 
Proof. We take absolute value of (4). Using Holder's inequality, we find that

$$
\begin{aligned}
& \left|\frac{1}{b-a} \int_{a}^{b} f(x) d x-\frac{f(a)+f(b)}{2}\right| \\
\leq & \frac{1}{b-a} \int_{a}^{b} \int_{a}^{b}|G(x, y)|\left|f^{\prime \prime}(y)\right| d y d x \\
= & \int_{a}^{b} \int_{a}^{x} \frac{(b-x)(y-a)}{(b-a)^{2}}\left|f^{\prime \prime}(y)\right| d y d x+\int_{a}^{b} \int_{x}^{b} \frac{(b-y)(x-a)}{(b-a)^{2}}\left|f^{\prime \prime}(y)\right| d y d x \\
\leq & \left(\int_{a}^{b} \int_{a}^{x} \frac{(b-x)^{p}(y-a)^{p}}{(b-a)^{2 p}} d y d x\right)^{\frac{1}{p}}\left(\int_{a}^{b} \int_{a}^{x}\left|f^{\prime \prime}(y)\right|^{q} d y d x\right)^{\frac{1}{q}} \\
& +\left(\int_{a}^{\frac{1}{p}} \frac{(b-y)^{p}(x-a)^{p}}{(b-a)^{2 p}} d y d x\right)^{b}\left(\int_{a}^{b}\left|f_{x}^{\prime \prime}(y)\right|^{q} d y d x\right)^{\frac{1}{q}} .
\end{aligned}
$$

If we calculate the above interals utilizing the convexity of $\left|f^{\prime \prime}(y)\right|^{q}$, from (11), then we obtain

$$
\begin{aligned}
& \left|\frac{1}{b-a} \int_{a}^{b} f(x) d x-\frac{f(a)+f(b)}{2}\right| \\
\leq & \left(\frac{(b-a)^{2}}{p+1} B(p+2, p+1)\right)^{\frac{1}{p}}\left(\frac{(b-a)^{3}}{2} \times \frac{2\left|f^{\prime \prime}(a)\right|^{q}+\left|f^{\prime \prime}(b)\right|^{q}}{3}\right)^{\frac{1}{q}} \\
& +\left(\frac{(b-a)^{2}}{p+1} B(p+1, p+2)\right)^{\frac{1}{p}}\left(\frac{(b-a)^{3}}{2} \times \frac{\left|f^{\prime \prime}(a)\right|^{q}+2\left|f^{\prime \prime}(b)\right|^{q}}{3}\right)^{\frac{1}{q}} .
\end{aligned}
$$

The proof is thus completed.

Theorem 2.4. Let $f: I^{\circ} \subseteq \mathbb{R} \rightarrow \mathbb{R}$ be a twice differentiable mapping on $I^{\circ}, a, b \in I^{\circ}$ with $a<b$. If $\left|f^{\prime \prime}\right|^{q}$ is convex on $[a, b], q \geq 1$, for all $y \in[a, b]$, then the following inequality holds:

$$
\left|\frac{1}{b-a} \int_{a}^{b} f(x) d x-\frac{f(a)+f(b)}{2}\right| \leq \frac{(b-a)^{2}}{12 \cdot 2^{\frac{1}{p}}}\left(\frac{\left|f^{\prime \prime}(a)\right|^{q}+\left|f^{\prime \prime}(b)\right|^{q}}{2}\right)^{\frac{1}{q}}
$$

where $\frac{1}{p}+\frac{1}{q}=1$. 
Proof. We take absolute value of (4). Because of $\frac{1}{p}+\frac{1}{q}=1, \frac{1}{p}+\frac{1}{q}$ can be written instead of 1. Using Holder's inequality, we find that

$$
\begin{aligned}
& \left|\frac{1}{b-a} \int_{a}^{b} f(x) d x-\frac{f(a)+f(b)}{2}\right| \\
\leq & \frac{1}{b-a} \int_{a}^{b} \int_{a}^{b}|G(x, y)|^{\frac{1}{p}+\frac{1}{q}}\left|f^{\prime \prime}(y)\right| d y d x \\
\leq & \frac{1}{b-a}\left(\int_{a}^{b} \int_{a}^{b}|G(x, y)| d y d x\right)^{\frac{1}{p}}\left(\int_{a}^{b} \int_{a}^{b}|G(x, y)|\left|f^{\prime \prime}(y)\right|^{q} d y d x\right)^{\frac{1}{q}} .
\end{aligned}
$$

We calculate the above integrals, respectively,

$$
\int_{a}^{b} \int_{a}^{b}|G(x, y)| d y d x=\frac{(b-a)^{3}}{24} \text {. }
$$

Since $\left|f^{\prime \prime}(t)\right|^{q}$ is convex on $[a, b]$, we get

$$
\int_{a}^{b} \int_{a}^{b}|G(x, y)|\left|f^{\prime \prime}(y)\right|^{q} d y d x=\frac{(b-a)^{3}}{12}\left(\frac{\left|f^{\prime \prime}(a)\right|^{q}+\left|f^{\prime \prime}(b)\right|^{q}}{2}\right) .
$$

Substituting the equalities (15) and (16) in (14), we obtain the inequality (13) which completes the proof.

\section{Applications to Numerical Integration}

We now consider applications of the integral inequalities developed in the previous section, to obtain estimates of composite quadrature rules which, it turns out have a markedly smaller error than that which may be obtained by the classical results.

Let $I_{n}: a=x_{0}<x_{1}<\ldots<x_{n-1}<x_{n}=b$ be a partition of the interval $[a, b]$, $\xi_{i} \in\left[x_{i}, x_{i+1}\right](i=0, \ldots, n-1)$ and consider the trapezoidal rule

$$
A_{T}\left(f, I_{n}\right)=\sum_{i=0}^{n-1}\left[\frac{f\left(x_{i}\right)+f\left(x_{i+1}\right)}{2}\right] h_{i}
$$

where $h_{i}=\left(x_{i+1}-x_{i}\right), i=0, \ldots, n-1$.

Theorem 3.1. Let $f: I^{\circ} \subseteq \mathbb{R} \rightarrow \mathbb{R}$ be a twice differentiable mapping on $I^{\circ}, a, b \in I^{\circ}$ with $a<b$. If $\left|f^{\prime \prime}\right|$ is convex on $[a, b]$, for all $y \in[a, b]$, then we have the representation

$$
\int_{a}^{b} f(x) d x=A_{T}\left(f, I_{n}\right)+R_{T}\left(f, I_{n}\right)
$$


where $A_{T}\left(f, I_{n}\right)$ is as defined in (17) and the remainder satisfies the astimations:

$$
\left|R_{T}\left(f, I_{n}\right)\right| \leq \frac{1}{12} \sum_{i=0}^{n-1}\left[\frac{\left|f^{\prime \prime}\left(x_{i}\right)\right|+\left|f^{\prime \prime}\left(x_{i+1}\right)\right|}{2}\right] h_{i}^{3}
$$

for $i=0, \ldots, n-1$.

Proof. Applying Theorem 2.1 on the interval $\left[x_{i}, x_{i+1}\right], i=0, \ldots, n-1$, we obtain

$$
\begin{aligned}
& \left|\int_{x_{i}}^{x_{i+1}} f(x) d x-\left[\frac{f\left(x_{i}\right)+f\left(x_{i+1}\right)}{2}\right] h_{i}\right| \\
& \leq \frac{1}{12}\left(\frac{\left|f^{\prime \prime}\left(x_{i}\right)\right|+\left|f^{\prime \prime}\left(x_{i+1}\right)\right|}{2}\right) h_{i}^{3}
\end{aligned}
$$

for $i=0, \ldots, n-1$.

Summing over $i$ from 0 to $n-1$ and using the triangle inequality we obtain the estimation (18).

Remark 3.1. If we consider the inequality (8), then we get the quadrature formula

$$
\int_{a}^{b} f(x) d x=A_{T}\left(f, I_{n}\right)+R_{T}\left(f, I_{n}\right)
$$

where $A_{T}\left(f, I_{n}\right)$ is trapezoidal formula and the remainder term satisfies the estimate

$$
\begin{aligned}
\left|R_{T}\left(f, I_{n}\right)\right| \leq & \frac{[B(p+2, p+1)+B(p+1, p+2)]^{\frac{1}{p}}}{(p+1)^{\frac{1}{p}}} \\
& \times \sum_{i=0}^{n-1}\left[\frac{\left|f^{\prime \prime}\left(x_{i}\right)\right|^{q}+\left|f^{\prime \prime}\left(x_{i+1}\right)\right|^{q}}{2}\right]^{\frac{1}{q}} h_{i}^{3} .
\end{aligned}
$$

Remark 3.2. If we consider the inequality (13), then we get the quadrature formula

$$
\int_{a}^{b} f(x) d x=A_{T}\left(f, I_{n}\right)+R_{T}\left(f, I_{n}\right)
$$

where $A_{T}\left(f, I_{n}\right)$ is trapezoidal formula and the remainder term satisfies the estimate

$$
\left|R_{T}\left(f, I_{n}\right)\right| \leq \frac{1}{12 \cdot 2^{\frac{1}{p}}} \sum_{i=0}^{n-1}\left[\frac{\left|f^{\prime \prime}\left(x_{i}\right)\right|^{q}+\left|f^{\prime \prime}\left(x_{i+1}\right)\right|^{q}}{2}\right]^{\frac{1}{q}} h_{i}^{3} .
$$




\section{Applications to Some Special Means}

Let us recall the following means:

(a) The arithmatic mean:

$$
A=A(a, b):=\frac{a+b}{2}, \quad a, b \geq 0
$$

(b) The Geometric mean:

$$
G=G(a, b):=\sqrt{a b}, \quad a, b \geq 0
$$

(c) The Harmonic mean:

$$
H=H(a, b):=\frac{2}{\frac{1}{a}+\frac{1}{b}}, \quad a, b>0
$$

(d) The Logarithmic mean:

$$
L=L(a, b):=\left\{\begin{array}{cl}
a & \text { if } a=b \\
\frac{b-a}{\ln b-\ln a} & \text { if } a \neq b
\end{array}, \quad a, b>0\right.
$$

(e) The Identric mean:

$$
I=I(a, b):=\left\{\begin{array}{cl}
a & \text { if } a=b \\
\frac{1}{e}\left(\frac{b^{b}}{a^{a}}\right)^{\frac{1}{b-a}} & \text { if } a \neq b
\end{array}, \quad a, b>0\right.
$$

(f) The $p$-logarithmic mean:

$$
L_{p}=L_{p}(a, b):=\left\{\begin{array}{cl}
a & \text { if } a=b \\
{\left[\frac{b^{p+1}-a^{p+1}}{(p+1)(b-a)}\right]^{\frac{1}{p}}} & \text { if } a \neq b
\end{array}, \quad a, b>0\right.
$$

where $p \in \mathbb{R} \backslash\{-1,0\}$.

The following simple relationships are known in literature

$$
H \leq G \leq L \leq I \leq A .
$$

It is also known that $L_{p}$ is monotonically increasing in $p \in \mathbb{R}$ with $L_{0}=I$ and $L_{-1}=L$. 3 .

(1) Consider the mapping $f:(0, \infty) \rightarrow \mathbb{R}, f(x)=x^{n}, x \in \mathbb{R}, n \in \mathbb{Z}$ and $|n(n-1)| \geq$

Then, we have, for $0<a<b$,

$$
\frac{1}{b-a} \int_{a}^{b} f(t) d t=L_{n}^{n}
$$

and

$$
\frac{f(a)+f(b)}{2}=A\left(a^{n}, b^{n}\right) .
$$

Using the inequality (6), we have the result:

$$
\left|L_{n}^{n}-A\left(a^{n}, b^{n}\right)\right| \leq|n(n-1)| \frac{(b-a)^{2}}{12} A\left(a^{n-2}, b^{n-2}\right) .
$$


Also, from (8), we get the inequality

$$
\begin{aligned}
\left|L_{n}^{n}-A\left(a^{n}, b^{n}\right)\right| \leq & |n(n-1)|[B(p+2, p+1)+B(p+1, p+2)]^{\frac{1}{p}} \\
& \times \frac{(b-a)^{2}}{(p+1)^{\frac{1}{p}}}\left[A\left(a^{q(n-2)}, b^{q(n-2)}\right)\right]^{\frac{1}{q}} .
\end{aligned}
$$

(2) Consider the mapping $f(x)=\frac{1}{x}, x \in[a, b] \subset(0, \infty)$.

Then, we have

$$
\frac{1}{b-a} \int_{a}^{b} f(t) d t=L_{-1}^{-1}=\frac{1}{L}
$$

and

$$
\frac{f(a)+f(b)}{2}=H^{-1}
$$

Using the inequality (6), we have the result:

$$
\left|L_{-1}^{-1}-H^{-1}\right| \leq \frac{(b-a)^{2}}{12} H^{-1}\left(a^{3}, b^{3}\right) .
$$

Also, from (13), we get the inequality

$$
\left|L_{-1}^{-1}-H^{-1}\right| \leq \frac{(b-a)^{2}}{12 \cdot 2^{\frac{1}{p}}}\left[H^{-1}\left(a^{3 q}, b^{3 q}\right)\right]^{\frac{1}{q}} .
$$

(3) Consider the mapping $f(x)=-\ln x, x \in[a, b] \subset(0, \infty)$.

Then, we have

$$
\frac{1}{b-a} \int_{a}^{b} f(t) d t=-\ln I(a, b)
$$

and

$$
\frac{f(a)+f(b)}{2}=-A(\ln a, \ln b) .
$$

Using the inequality (6), we have the result:

$$
|A(\ln a, \ln b)-\ln I(a, b)| \leq \frac{(b-a)^{2}}{12} H^{-1}\left(a^{2}, b^{2}\right) .
$$

Also, from (13), we get the inequality

$$
|A(\ln a, \ln b)-\ln I(a, b)| \leq \frac{(b-a)^{2}}{12 \cdot 2^{\frac{1}{p}}}\left[H^{-1}\left(a^{2 q}, b^{2 q}\right)\right]^{\frac{1}{q}} .
$$




\section{References}

[1] S. S. Dragomir and R.P. Agarwal, Two inequalities for differentiable mappings and applications to special means of real numbers and to trapezoidal formula, Appl. Math. lett., 11(5) (1998), 91-95.

[2] S. S. Dragomir and C. E. M. Pearce, Selected Topics on Hermite-Hadamard Inequalities and Applications, RGMIA Monographs, Victoria University, 2000.

[3] S. Hussain, M.I. Bhatti and M. Iqbal, Hadamard-type inequalities for s-convex functions I, Punjab Univ. Jour. of Math., Vol.41, pp:51-60, (2009).

[4] U.S. Kırmac1, Inequalities for differentiable mappings and applications to special means of real numbers and to midpoint formula, Appl. Math. Comp, 147 (2004), 137-146.

[5] J. Pečarić, F. Proschan and Y.L. Tong, Convex functions, partial ordering and statistical applications, Academic Press, New York, 1991.

[6] C.E.M. Pearce and J. Pěcarić, Inequalities for differentiable mappings with application to special means and quadrature formulae, Appl. Math. Lett., 13(2) (2000), 51-55.

[7] M. Z. Sarikaya, A. Saglam and H. Yıldırım, New inequalities of Hermite-Hadamard type for functions whose second derivatives absolute values are convex and quasi-convex, (submitted for publication) arXiv:1005.0451.

[8] M. Z. Sarikaya and N. Aktan, On the generalization of some integral inequalities and their applications, Mathematical and Computer Modelling, 54 (2011), 2175-2182.

[9] M. Z. Sarikaya, A. Saglam and H. Yıldırım, Some new integral inequalities for twice differentiable convex mappings, (submitted for publication) arXiv:1005.0453v1.

[10] K-L. Tseng, G-S. Yang and K-C. Hsu, Some inequalities for differentiable mappings and applications to Fejer inequality and weighted trapozidal formula, Taiwanese J. Math. 15(4), pp:1737-1747, 2011.

[11] G.S. Yang, D.Y. Hwang, K.L. Tseng, Some inequalities for differentiable convex and concave mappings, Comput. Math. Appl. 47 (2004), 207-216.

[12] C.-L. Wang, X.-H. Wang, On an extension of Hadamard inequality for convex functions, Chin. Ann. Math. 3 (1982) 567-570.

[13] S. Wasowicz and A. Witkonski, On some inequality of Hermite-Hadamard type, Opuscula Math. 32(2), (2012), pp:591-600.

[14] B-Y, Xi and F. Qi, Some Hermite-Hadamard type inequalities for differentiable convex functions and applications, Hacet. J. Math. Stat.. 42(3), 243-257 (2013).

[15] B-Y, Xi and F. Qi, Hermite-Hadamard type inequalities for functions whose derivatives are of convexities, Nonlinear Funct. Anal. Appl.. 18(2), 163-176 (2013). 